\title{
Reaction boundary between akimotoite and ringwoodite + stishovite in $\mathrm{MgSiO}_{3}$
}

\author{
Shigeaki Ono ${ }^{1}$ (D) Takumi Kikegawa ${ }^{2} \cdot$ Yuji Higo $^{3}$
}

Received: 4 October 2016 / Accepted: 27 December 2016 / Published online: 13 January 2017

(C) The Author(s) 2017. This article is published with open access at Springerlink.com

\begin{abstract}
The phase boundary between akimotoite and ringwoodite + stishovite in $\mathrm{MgSiO}_{3}$ was determined using a multi-anvil high-pressure apparatus and synchrotron X-ray radiation. The phase relation was determined by observing the recovered samples using an electron microprobe analyzer. Experimental pressures were monitored by the in situ powdered X-ray diffraction data of gold, which was put in the sample chamber. The reaction boundary between akimotoite and ringwoodite + stishovite was found to occur at $P(\mathrm{GPa})=22.0-0.0012 \times T(\mathrm{~K})$. The pressure dependence of the slope of the reaction boundary, $\mathrm{d} P / \mathrm{d} T$, determined in our study was smaller than that determined by Gasparik ( $\mathbf{J}$ Geophys Res 95:15751-15769, 1990). The triple point of ringwoodite + stishovite-wadsleyite + stishovite-akimotoite estimated in our study was at $\sim 20 \mathrm{GPa}$ and $\sim 1700 \mathrm{~K}$.
\end{abstract}

Keywords Akimotoite $\cdot$ Ringwoodite $\cdot$ Stishovite $\cdot$ High pressure

\section{Introduction}

Knowledge of the phase relations in $\mathrm{MgSiO}_{3}$ is critical for understanding the layered structure, dynamics, and

Shigeaki Ono

sono@jamstec.go.jp

1 Research and Development Center for Ocean Drilling Science, Japan Agency for Marine-Earth Science and Technology, 2-15 Natsushima-cho, Yokosuka-shi, Kanagawa 237-0061, Japan

2 High Energy Acceleration Research Organization, 1-1 Oho, Tsukuba 305-0801, Japan

3 Japan Synchrotron Radiation Research Institute, Sayo-cho, Sayo-gun, Hyogo 679-5198, Japan evolution of the Earth's mantle. It is widely accepted that the seismic discontinuities at 410 and $660 \mathrm{~km}$ depth (Dziewonski and Anderson 1981) are due to the phase transition from olivine to wadsleyite and from ringwoodite to bridgmanite + ferro-periclase in $(\mathrm{Mg}, \mathrm{Fe})_{2} \mathrm{SiO}_{4}$ (e.g., Katsura and Ito 1989; Ito and Takahashi 1989). For the natural mantle composition, such as peridotite, the primary minerals are $(\mathrm{Mg}, \mathrm{Fe})_{2} \mathrm{SiO}_{4}$-related phases, and the secondary minerals are pyroxene and garnet in the system $(\mathrm{Mg}, \mathrm{Fe}) \mathrm{SiO}_{3}-(\mathrm{Mg}$, $\mathrm{Fe})_{3} \mathrm{Al}_{2} \mathrm{Si}_{3} \mathrm{O}_{12} . \mathrm{MgSiO}_{3}$ is the end component in a complicated composition of pyroxene. According to a previous study (Gasparik 1990), wadsleyite and stishovite change to akimotoite $\left(\mathrm{MgSiO}_{3}\right)$ with increasing pressure along the geotherm. In contrast, Sawamoto (1987) showed that wadsleyite and stishovite change into ringwoodite $\left(\mathrm{Mg}_{2} \mathrm{SiO}_{4}\right)$ and stishovite before the formation of akimotoite. This discrepancy is attributed to the estimation of the phase boundary between ringwoodite + stishovite and akimotoite. As this reaction can be observed in the relatively low-temperature region, the previous experiments (Sawamoto 1987; Gasparik 1990) might have involved a significant uncertainty related to the effects of the reaction kinetics.

In our study, the use of a multi-anvil high-pressure system combined with a synchrotron radiation source enabled the acquisition of precise data for experimental pressures from samples under high-pressure and high-temperature conditions. To determine more accurate experimental pressures, we measured powder X-ray diffraction (XRD) data of gold, which was used as the pressure standard, in the sample chamber. Herein, we report on the disputed issue of the phase boundary between ringwoodite + stishovite and akimotoite in $\mathrm{MgSiO}_{3}$, and a revised pressure-temperature $(\mathrm{P}-\mathrm{T})$ phase diagram in $\mathrm{MgSiO}_{3}$ based on our data will be suggested. 


\section{Methods}

A synthetic gel was used to produce a reactive and homogeneous starting material (Ono and Yasuda 1996). The chemical composition of the synthesized gel was $\mathrm{MgSiO}_{3}$. High-pressure XRD experiments were performed using a multi-anvil high-pressure apparatus comprised of eight cubic anvils made of tungsten carbide (WC) with truncations of 1.5 or $2.0 \mathrm{~mm}$. The cubic anvil assembly was compressed using "Max III" and "SPEED 1500" high-pressure apparatus, and was installed at the synchrotron facilities of KEK and SPring- 8 in Japan. The system was comprised of a 700-ton (at KEK) or 1500-ton (at SPring-8) multi-anvil press equipped with an energy-dispersive X-ray diffractometer system with a germanium solid-state detector. The incident beam was white $\mathrm{X}$-rays. The width of both the incident and diffracted X-ray beams was $50 \mu \mathrm{m}$, and the diffracted angle was $2 \theta=6.0^{\circ}$. A Cr-doped $\mathrm{MgO}$ pressure medium was placed at the center of the WC cubic anvil assembly and enclosed in pyrophyllite gaskets. A cylindrical heater made of $\mathrm{TiB}_{2}$ (Ono 2016) was inserted into the octahedral pressure medium and enclosed in the $\mathrm{ZrO}_{2}$ sleeve for thermal insulation. Two $\mathrm{MgO}$ rods of $1.0 \mathrm{~mm}$ diameter extended from both edges of the octahedron to the heater through the pressure medium and the $\mathrm{ZrO}_{2}$ sleeve, and provided a path for the incident and diffracted X-ray beams. Details of the cell assembly are elsewhere (Ono et al. 2011, 2013). The powdered sample and gold, which was used as a pressure standard, were loaded directly into the heater, which also served as the sample capsule. The sample temperature was monitored using a $\mathrm{W}_{97} \mathrm{Re}_{3}-\mathrm{W}_{75} \mathrm{Re}_{25}$ thermocouple inserted at the center of the sample chamber. No correction was made to counter the effect of pressure on the thermocouple EMF. The typical temperature fluctuation during heating was around $\pm 5^{\circ} \mathrm{C}$. The $\mathrm{X}$-ray measurements of the sample were taken close to the thermocouple junction $(<100$ microns $)$; therefore, the temperature gradient between the X-ray position and the thermocouple junction was kept within $50{ }^{\circ} \mathrm{C}$. The pressure was determined from the unit cell volume of gold using the gold equation of state (EOS) (Dorogokupets and Dewaele 2007). The typical uncertainty in pressure was within 0.2 GPa. Dorogokupets and Dewaele (2007) established internally consistent EOSs of $\mathrm{Au}, \mathrm{MgO}, \mathrm{Pt}, \mathrm{B} 1-\mathrm{NaCl}$, and $\mathrm{B} 2-\mathrm{NaCl}$. Therefore, their pressure scale has an advantage to compare experimental results using different materials for the pressure scale.

Pressure was applied to the sample by generating an anvil load from the desired oil pressure in the press. Before heating, the peaks of gold were broad which was induced by the effect of the accumulated differential stress during compression. The sample was then quickly heated until it reached the desired temperature for a given oil pressure. The typical rate of temperature increase was $\sim 200 \mathrm{~K} / \mathrm{min}$.
As the temperature increased, the peaks of gold became intense around $1000 \mathrm{~K}$. This indicated that the recrystallization of gold had started and that the accumulated stress was gradually released. After the desired temperature was reached, the measurement of pressure started and continued until the temperature quenching. The temperature was maintained for 1.7-4.0 h. A significant deviation in the intensity ratio of the diffraction peaks of gold from the ideal data in the reference database was observed at higher temperatures because of grain growth of gold crystals. However, pressure could also be monitored during heating. A significant change in the experimental pressure, which was induced by the deformation of the pressure medium after reaching the desired temperature, was not confirmed. The typical pressure fluctuation was $\sim 0.3 \mathrm{GPa}$, which was similar to that found in our previous studies (Ono et al. 2011, 2013). At the end of the experimental runs, the sample was quenched by turning off the electrical power, which resulted in the temperature dropping to below $400 \mathrm{~K}$ within a period of $3 \mathrm{~s}$, after which the pressure decreased slowly and the sample could be removed. After quenching, the samples were polished for investigation with an electron microprobe analyzer (JXA-8500F, JEOL). The stable phase in each experimental run was determined by the chemical composition of phases from this analysis.

\section{Results}

The experimental runs were performed at pressures between 19 and $21 \mathrm{GPa}$ (Table 1). Figure 1 shows the

Table 1 Experimental conditions and results

\begin{tabular}{lllll}
\hline$T(\mathrm{~K})$ & $P(\mathrm{GPa})$ & $a(\AA)$ & Time (h) & Phases present \\
\hline 1100 & $21.2(1)$ & $3.976(1)$ & 3.0 & Akimotoite \\
1150 & $19.2(2)$ & $3.989(1)$ & 3.0 & Ringwoodite + Stishovite \\
1150 & $20.0(1)$ & $3.984(1)$ & 4.0 & Ringwoodite + Stishovite \\
1150 & $20.5(1)$ & $3.982(1)$ & 3.0 & Ringwoodite + Stishovite \\
1150 & $20.8(5)$ & $3.980(3)$ & 3.0 & Akimotoite \\
1200 & $19.1(1)$ & $3.991(1)$ & 3.0 & Ringwoodite + Stishovite \\
1200 & $20.8(1)$ & $3.982(1)$ & 2.0 & Akimotoite \\
1250 & $19.0(2)$ & $3.994(1)$ & 3.0 & Ringwoodite + Stishovite \\
1300 & $19.9(1)$ & $3.991(1)$ & 2.0 & Ringwoodite + Stishovite \\
1300 & $20.5(1)$ & $3.987(1)$ & 2.0 & Akimotoite \\
1300 & $20.7(1)$ & $3.986(1)$ & 2.0 & Akimotoite \\
1350 & $19.9(1)$ & $3.993(1)$ & 3.0 & Ringwoodite + Stishovite \\
1350 & $20.4(1)$ & $3.990(1)$ & 2.0 & Ringwoodite + Stishovite \\
1400 & $20.4(1)$ & $3.992(1)$ & 1.7 & Akimotoite \\
1450 & $20.2(1)$ & $3.995(1)$ & 3.0 & Ringwoodite + Stishovite \\
\hline
\end{tabular}

$\mathrm{T}, \mathrm{P}$, and $a$ are the temperature, pressure, and lattice parameter of gold, respectively 


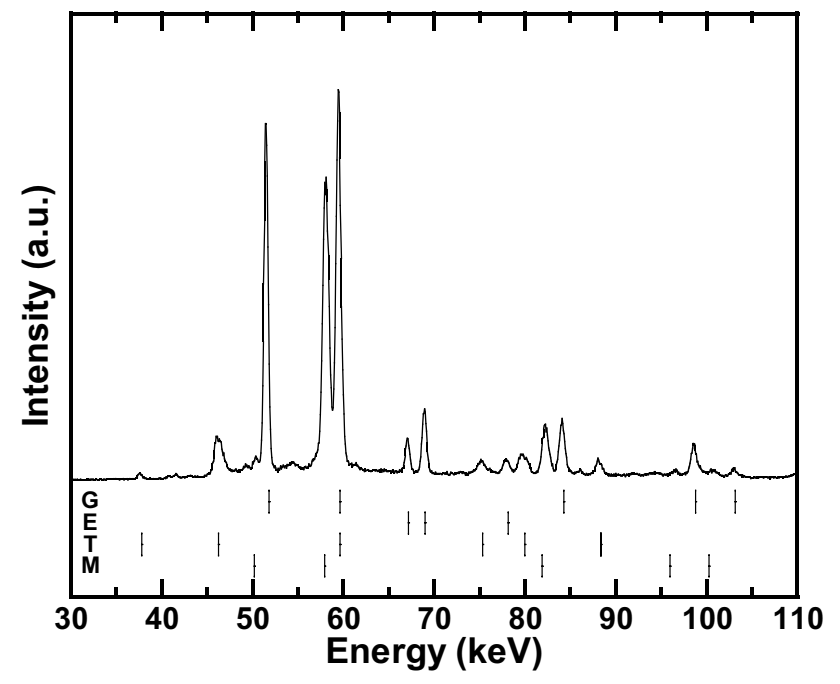

Fig. 1 Example of the observed XRD pattern of the sample. Data were acquired at $P=20.2 \mathrm{GPa}$ and $T=1450 \mathrm{~K}$. The vertical bars denote the calculated positions of the diffraction and emission lines of each phase. Key to label abbreviations: $G$ gold (Au), $E$ emission of gold fluorescence, $T \mathrm{TiB}_{2}, M$ periclase $(\mathrm{MgO})$

typical XRD data acquired at $20.2 \mathrm{GPa}$ and $1450 \mathrm{~K}$. The powder XRD peaks of gold and its fluorescence emissions were intense enough to calculate the experimental pressures. In addition to the peaks from gold, some peaks from $\mathrm{TiB}_{2}$ and $\mathrm{MgO}$ were observed. $\mathrm{TiB}_{2}$ and $\mathrm{MgO}$ were used as the heater and the rods for the path for the X-ray beam, respectively. Although accurate experimental pressures were calculated, the peaks from the sample could not be identified, because the intense peaks from gold,
$\mathrm{TiB}_{2}$ and $\mathrm{MgO}$ obscured the weak peaks from the sample. Figure 2 shows typical images of the quenched sample obtained using the electron microprobe analyzer. At pressures below the reaction boundary, the $\mathrm{MgSiO}_{3}$ starting material changed to the $\mathrm{Mg}$-rich and silica phases (Fig. 2a). The chemical compositions of the light- and dark-gray phases were pure $\mathrm{SiO}_{2}$ and $\mathrm{Mg}_{2} \mathrm{SiO}_{4}$, respectively. As the experimental pressure in each case was higher than for the boundaries of the wadsleyite-ringwoodite and coesite-stishovite, the $\mathrm{Mg}_{2} \mathrm{SiO}_{4}$ and $\mathrm{SiO}_{2}$ phases were judged as ringwoodite and stishovite. In contrast, the starting material changed to a single phase of akimotoite, which was the dark-gray phase $\left(\mathrm{MgSiO}_{3}\right)$, under pressures higher than the reaction boundary (Fig. 2b). In this study, it was easy to identify the phase relation by chemical analysis of the quenched sample containing the gold powder used for pressure monitoring.

We performed 15 experimental runs to investigate the reaction boundary between ringwoodite + stishovite and akimotoite (Table 1). The pressure-temperature (P-T) conditions of the acquired XRD patterns of gold and the stable phases identified from the quenched samples are shown in Fig. 3. The experimental pressures were lower and higher than those corresponding to the stability fields of bridgmanite and wadsleyite, respectively. The gradient of $\mathrm{d} P / \mathrm{d} T$ of the reaction boundary determined in our study was negative. The transition boundary shown in Fig. 3 is represented by the following linear equation:

$P(\mathrm{GPa})=22.0(4)-0.0012(6) T(\mathrm{~K})$.

The boundary determined in this study was in good agreement with that reported by Sawamoto (1987). In

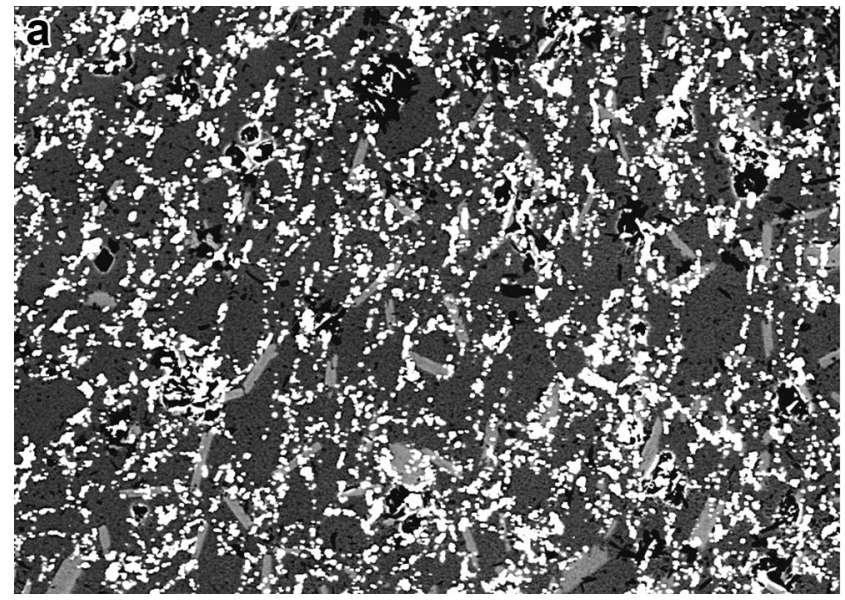

Fig. 2 Examples of compositional images in back-scattered electron microphotographs of the quenched samples product. a White parts are the gold phase used as the pressure reference. Dark and light grays are $\mathrm{Mg}_{2} \mathrm{SiO}_{4}$ and $\mathrm{SiO}_{2}$, respectively. Black parts are the void filled with the epoxy. The horizontal scale is $200 \mu \mathrm{m}$. The experi-

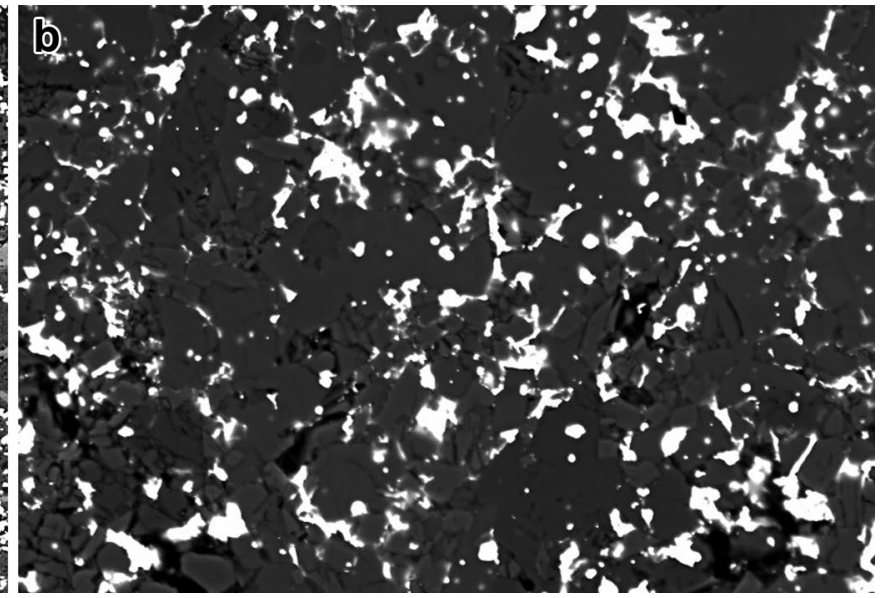

mental condition was $1150 \mathrm{~K}$ and $20.5 \mathrm{GPa}$. b White, gray, and black parts are gold, $\mathrm{MgSiO}_{3}$, and the void, respectively. The small contrast in gray parts is the difference in the crystal orientation. The horizontal scale is $90 \mu \mathrm{m}$. The experimental condition was $1300 \mathrm{~K}$ and 20.7 $\mathrm{GPa}$ 


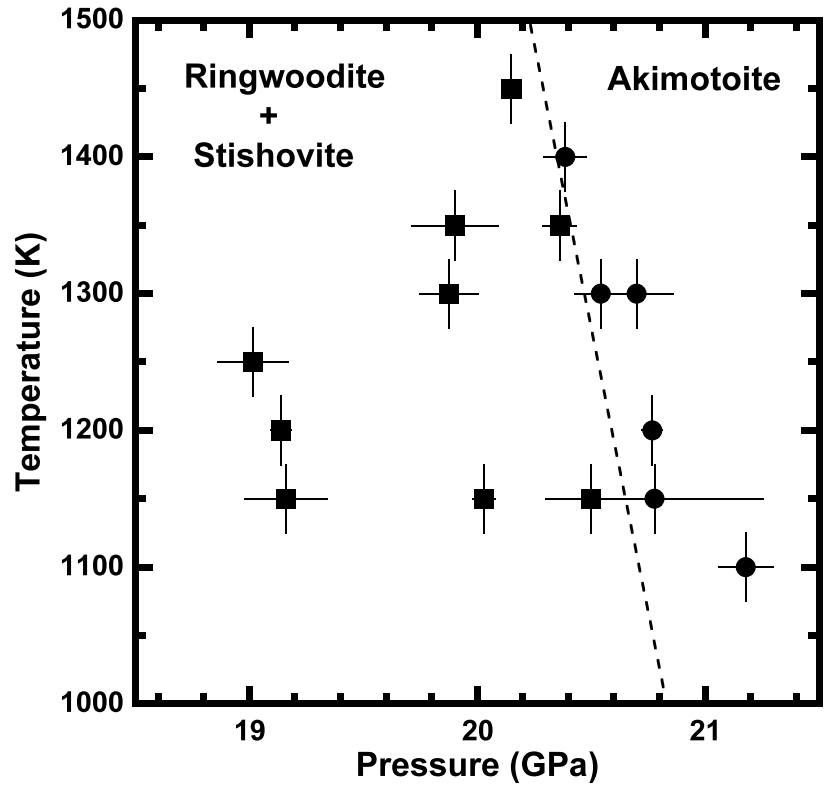

Fig. 3 Experimental results and reaction boundary between ringwoodite + stishovite and akimotoite in $\mathrm{MgSiO}_{3}$. Squares and circles denote the stability conditions of ringwoodite + stishovite and akimotoite, respectively. The dashed line shows the reaction boundary determined in this study

contrast, the $\mathrm{d} P / \mathrm{d} T$ slope reported by Gasparik (1990) was inconsistent with the slopes determined in this study and by Sawamoto (1987).

We used the EOS of gold by Dorogokupet and Dewaele (2007). This EOS has been repeatedly investigated in the previous experimental and theoretical studies (e.g., Jamieson et al. 1982; Anderson et al. 1989; Holzapfel et al. 2001; Takemura 2001, 2007; Okube et al. 2002; Shim et al. 2002; Boettger 2003; Dewaele et al. 2004; Greeff et al. 2004; Souvatzis et al. 2006; Dorogokupet and; Dewaele 2007; Fei et al. 2007; Holzapfel and Nicol 2007). However, the validity of the EOS of gold is still questionable. Therefore, it is accepted that the uncertainty of experimental pressure might be $<10 \%$.

\section{Discussion}

A comparison between our study and previous determinations of the $\mathrm{d} P / \mathrm{d} T$ slope is shown in Fig. 4. The pressure of the reaction boundary around $1200 \mathrm{~K}$ that was determined in our study was in general agreement with values reported in the previous studies (Ito and Navrotsky 1985; Kanzaki 1987; Sawamoto 1987; Gasparik 1990; Stixrude and Lithgow-Bertelloni 2011). However, as indicated earlier, the $\mathrm{d} P / \mathrm{d} T$ slope reported by Gasparik (1990) was inconsistent with the slopes reported by Sawamoto (1987) and our study. Several possible explanations for this discrepancy

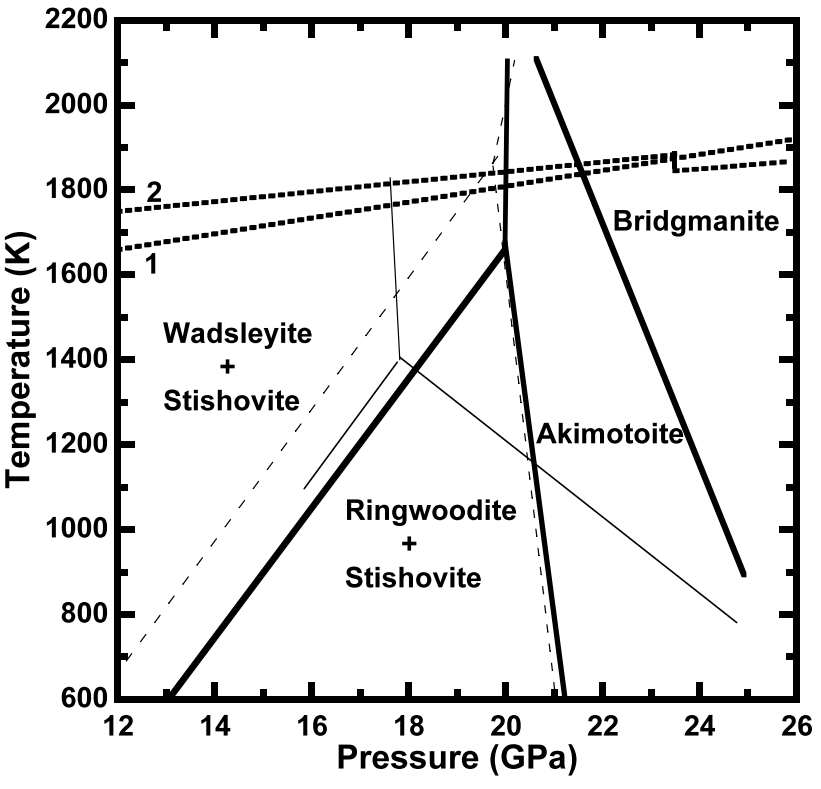

Fig. 4 Comparison of phase diagram of $\mathrm{MgSiO}_{3}$ between our study and previous studies at $12-25 \mathrm{GPa}$ and $600-2200 \mathrm{~K}$. The thick lines are estimates in our study. The boundaries between wadsleyite and ringwoodite and between akimotoite and bridgmanite are based on the works of Suzuki et al. (2000) and Ono et al. (2001), respectively, revised using the EOS of gold (Dorogokupets and Dewaele 2007). The boundary between wadsleyite + stishovite and akimotoite was not constrained based solely on our data. The thin dashed and solid lines are phase boundaries determined by Sawamoto (1987) and Gasparik (1990), respectively. Thick dotted lines are the geotherms: 1 Brown and Shankland (1981) and 2 Ono (2008)

can be proposed. First, it is known that the pressure calibration used in Gasparik's study might involve uncertainty. Gasparik (1990) used the conventional method for pressure calibration in the quench experiment. The efficiency of the pressure generation of the high-pressure apparatus was calibrated using several fixed pressure points before the experiments. It is accepted that this method can lead to a significant error in the pressure determination. In contrast, we used in situ monitoring of the experimental pressure using the synchrotron XRD method.

Second, the chemical reaction kinetics often affect the determination of the reaction boundary. It is known that the effect of the reaction kinetics is significant at low temperatures. In general, it is difficult to identify stable phases at low-temperature conditions, and errors in such identification might lead to the discrepancies between the different $\mathrm{d} P / \mathrm{d} T$ slopes. To enhance the chemical reaction, our staring material was the gel, which was a reactive material compared with the oxide mixtures.

Third, a difference in the chemical composition of the starting materials might induce the discrepancy. The oxide mixtures, including a flux that was expected to enhance the chemical reaction, were used as the starting material by 
Gasparik (1990). In contrast, pure $\mathrm{MgSiO}_{3}$ was used as the starting material by Sawamoto (1987) and in the present study. The fluxes, which were lead oxide or lead fluoride (Gasparik 1990), might affect the stability of each phase.

According to the phase diagram of $\mathrm{MgSiO}_{3}$, the location of the triple point of wadsleyite + stishovite-ringwoodite + stishovite-akimotoite is important for predicting the reaction sequence along the geotherm in the Earth's mantle. If the temperature of the triple point is lower than the geotherm (i.e., Gasparik 1990), the wadsleyite + shishovite mixture changes directly into akimotoite as the pressure increases. When the reaction boundary determined in our study is combined with the phase boundary between wadsleyite and ringwoodite determined by Suzuki et al. (2000) using the in situ XRD method, the estimated triple point locates at $1700 \pm 100 \mathrm{~K}$ and $20 \pm 1 \mathrm{GPa}$, which is $2 \mathrm{GPa}$ and $300 \mathrm{~K}$ higher than that reported by Gasparik (1990). The phase boundary estimated in our study is in agreement with that reported by Sawamoto (1987); however, the temperature of the triple point of our study is $200 \mathrm{~K}$ lower than that determined by Sawamoto (1987). A probable explanation for this discrepancy is that the experimental conditions of Sawamoto (1987) were an overestimate for temperature and an underestimate for pressure. In the work by Sawamoto (1987), the experimental pressure was calibrated at room temperature using the pressure fixed points of several phase transitions, and no temperature correction was made for the effect of the anvil surface temperature. The triple point estimated by our study was located at a temperature slightly lower than the geotherm (Brown and Shankland 1981; Ono 2008). The phase boundary between wadsleyite and ringwoodite has been investigated repeatedly in the previous studies (e.g., Katsura and Ito, 1898; Akaogi et al. 1989; Inoue et al. 2006). If these steeper Clapeyron slopes, which might be less accurate than that of Suzuki et al. (2000), are used to estimate the triple point, this triple point would move to a higher temperature region.

Acknowledgements The synchrotron radiation experiments were performed at the NE7A, KEK (Proposal No. 2013G512) and BL04B1, SPring-8 (Proposal Nos. 2013A1201 and 2014B1161).

Open Access This article is distributed under the terms of the Creative Commons Attribution 4.0 International License (http:// creativecommons.org/licenses/by/4.0/), which permits unrestricted use, distribution, and reproduction in any medium, provided you give appropriate credit to the original author(s) and the source, provide a link to the Creative Commons license, and indicate if changes were made.

\section{References}

Akaogi M, Ito E, Navrotsky A (1989) Olivine-modified spinel-spinel transitions in the system $\mathrm{Mg}_{2} \mathrm{SiO}_{4}-\mathrm{Fe}_{2} \mathrm{SiO}_{4}$ : calorimetric measurements, thermochemical calculation, and geophysical application. J Geophys Res 94:15671-15685

Anderson OL, Isaak DG, Yamamoto S (1989) Anharmonicity and the equation of state for gold. J Appl Phys 65:1534-1543

Boettger JC (2003) Theoretical extension of the gold pressure calibration standard beyond 3 Mbars. Phys Rev B 67:174107

Brown JM, Shankland TJ (1981) Thermodynamic parameters in the Earth as determined from seismic profiles. Geophys J R Astr Soc 66:579-596

Dewaele A, Loubeyre P, Mezouar M (2004) Compression curves of transition metals in the Mbar range: experiments and projector augmented-wave calculations. Phys Rev B 70:094112

Dorogokupets PI, Dewaele A (2007) Equations of state of $\mathrm{MgO}, \mathrm{Au}$, $\mathrm{Pt}, \mathrm{NaCl}-\mathrm{B} 1$, and NaCl-B2: Internally consistent high-temperature pressure scales. High Press Res 27:431-446

Dziewonski AM, Anderson DL (1981) Preliminary refence earth model. Phys Earth Planet Inter 25:297-356

Fei Y, Ricolleau A, Frank M, Mibe K, Shen G, Prakapenka V (2007) Toward an internally consistent pressure scale. Proc Nat Acad Sci USA 104:9182-9186

Gasparik T (1990) Phase relations in the transition zone. J Geophys Res 95:15751-15769

Greeff CW, Graf MJ (2004) Lattice dynamics and the high-pressure equation of state of Au. Phys Rev B 69:054107

Holzapfel WB, Nicol M (2007) Refined equations of state for Cu, Ag, and $\mathrm{Au}$ in the sub-TPa region. High Press Res 27:377-392

Holzapfel WB, Hartwig M, Sievers W (2001) Equations of state for $\mathrm{Cu}, \mathrm{Ag}$, and $\mathrm{Au}$ for wide ranges in temperature and pressure up to $500 \mathrm{GPa}$ and above. J Phys Chem Ref Data 30:515-529

Inoue T, Irifune T, Higo Y, Sanehira T, Sueda Y, Yamada A, Shinmei T, Yamazaki D, Ando J, Funakoshi K, Utsumi W (2006) The phase boundary between wadsleyite and ringwoodite in $\mathrm{Mg}_{2} \mathrm{SiO}_{4}$ determined by in situ X-ray diffraction. Phys Chem Minerals 33:106-114

Ito E, Navrotsky A (1985) $\mathrm{MgSiO}_{3}$ ilmenite: calorimetry, phase equilibria, and decomposition at atmospheric pressure. Am Mineral 70:1020-1026

Ito E, Takahashi E (1989) Postspinel transitions in the system $\mathrm{Mg}_{2} \mathrm{SiO}_{4}-\mathrm{Fe}_{2} \mathrm{SiO}_{4}$ and some geophysical implications. J Geophy Res 94:10637-10646

Jamieson JC, Fritz JN, Manghnani MH (1982) Pressure measurement at high temperature in X-ray diffraction studies: gold as a primary standard. In: Akimoto S, Manghnani MH (eds) High-pressure research in geophysics. Center for Academic Publishing, Tokyo, pp 27-48

Kanzaki M (1987) Ultrahigh-pressure phase relations in the system $\mathrm{Mg}_{4} \mathrm{Si}_{4} \mathrm{O}_{12}-\mathrm{Mg}_{3} \mathrm{Al}_{2} \mathrm{Si}_{3} \mathrm{O}_{12}$. Phys Earth Planet Inter 49:168-175

Katsura T, Ito $\mathrm{E}$ (1989) The system $\mathrm{Mg}_{2} \mathrm{SiO}_{4}-\mathrm{Fe}_{2} \mathrm{SiO}_{4}$ at high pressures and temperatures: precise determination of stabilities of olivine, modified spinel, and spinel. J Geophys Res 94:15663-15670

Okube M, Yoshiasa A, Ohtaka O, Fukui H, Katayama Y, Utsumi W (2002) Anharmonicity of gold under high-pressure and hightemperature. Solid State Commun 121:235-239

Ono S (2008) Experimental constraints on the temperature profile in the lower mantle. Phys Earth Planet Inter 170:267-273

Ono S (2016) Titanium boride equation of state determined by in-situ $\mathrm{X}$-ray diffraction. Heliyon 2:e00220

Ono S, Yasuda A (1996) Compositional change of majoritic garnet in a MORB composition from 7 to $17 \mathrm{GPa}$ and 1400 to $1600{ }^{\circ} \mathrm{C}$. Phys Earth Planet Inter 96:171-179

Ono S, Katsura T, Ito E, Kanzaki M, Yoneda A, Walter MJ, Urakawa S, Utsumi W, Funakoshi K (2001) In situ observation of ilmenite-perovskite phase transition in $\mathrm{MgSiO}_{3}$ using synchrotron radiation. Geophys Res Lett 28:835-838 
Ono S, Kikegawa T, Higo Y (2011) In situ observation of a garnet-perovskite transition in $\mathrm{CaGeO}_{3}$. Phys Chem Minerals 38:735-740

Ono S, Kikegawa T, Higo Y (2013) In situ observation of a phase transition in $\mathrm{Fe}_{2} \mathrm{SiO}_{4}$ at high pressure and high temperature. Phys Chem Minerals 40:811-816

Sawamoto $\mathrm{H}$ (1987) Phase diagram of $\mathrm{MgSiO}_{3}$ at pressures up to 24 $\mathrm{GPa}$ and temperatures up to $2200^{\circ} \mathrm{C}$ : phase stability and properties of tetragonal garnet. In Manfhnani MH, Syono Y (eds) High-pressure research in mineral physics. Terra-pub, Tokyo. pp 209-219

Shim S-H, Duffy TS, Takemura K (2002) Equation of state of gold and its application to the phase boundaries near $660 \mathrm{~km}$ depth in Earth's mantle. Earth Planet Sci Lett 203:729-739
Souvatzis P, Delin A, Eriksson O (2006) Calculation of the equation of state of fcc Au from first principles. Phys Rev B 73:054110

Stixrude L, Lithqow-Bertelloni C (2011) Thermodynamics of mantle minerals-II. Phase equilibria. Geophys J Int 184:1180-1213

Suzuki A, Ohtani E, Morishima H, Kubo T, Kanbe Y, Kondo T, Okada T, Terasaki H, Kato T, Kikegawa T (2000) In situ determination of the boundary between wadsleyite and ringwoodite in $\mathrm{Mg}_{2} \mathrm{SiO}_{4}$. Geophys Res Lett 27:803-806

Takemura K (2001) Evaluation of the hydrostaticity of a helium-pressure medium with powder X-ray diffraction techniques. J Appl Phys 89:662-668

Takemura K (2007) Pressure scales and hydrostaticity. High Press Res $27: 465-472$ 